\title{
MARIE-NiCOLE GRAS
}

\section{Nombre de classes, unités et bases d'entiers des extensions cubiques cycliques de $Q$}

\author{
Mémoires de la S. M. F., tome 37 (1974), p. 101-106 \\ <http://www.numdam.org/item?id=MSMF_1974_37_101_0>
}

(C) Mémoires de la S. M. F., 1974, tous droits réservés.

L'accès aux archives de la revue « Mémoires de la S. M. F. » (http://smf. emath.fr/Publications/Memoires/Presentation.html) implique l'accord avec les conditions générales d'utilisation (http://www.numdam.org/conditions). Toute utilisation commerciale ou impression systématique est constitutive d'une infraction pénale. Toute copie ou impression de ce fichier doit contenir la présente mention de copyright.

\section{Numdam}


Journées arithmétiques

Bull. Soc. math. France,

Mémoire 37, 1974, p. 101-106

\section{NOMBRE DE CLASSES, UNITES ET BASES D'ENTIERS \\ DES EXTENSIONS CUBIQUES CYCLIQUES DE $Q$}

par

Miarie Nicole GRAS

-:-::-:-

Soit $\mathrm{K}$ un corps cubique cyclique. Nous établissons un algorithme permettant de déterminer le nombre de classes $h$ de $K$ et un générateur $\epsilon$ du groupe $E$ des unités de norme 1 de $K$, à partir de la seule connaissance d'un générateur du groupe des unités cyclotomiques de $\mathrm{K}$. La connaissance du groupe des unités permet aussi une étude de l'existence des bases d'entiers de la forme $\left\{1, \vartheta, \vartheta^{2}\right\}$. L'utilisation d'un ordinateur nous a permis de dresser des tables, dont une donnant le nombre de classes et les unités pour les corps de conducteur inférieur à 4000 . Ces méthodes faisant l'objet de publications ([2] et [3]), nous ne donnerons ici que les principaux résultats.

\section{NOTATIONS ET RAPPELS.}

Soit $\mathrm{K}$ une extension cubique cyclique de $Q$ de conducteur $\mathrm{m}$; son discriminant est égal à $\mathrm{m}^{2}$. Soit $\mathrm{A}$ l'anneau des entiers de $\mathrm{K}$ et soit $\sigma$ un générateur de $\mathrm{G}=\mathrm{Gal}(\mathrm{K} / \mathbb{Q})$. Soit $\mathrm{n}$ le nombre de diviseurs premiers du discriminant de $K / Q^{\circ}$; on posera $m=p_{1} \ldots p_{n}$, en convenant que $p_{n}=9$ si 3 est ramifié dans $\mathrm{K} / \Phi$. Avec ces notations, $m$ se met dans tous les cas sous la forme $m=\frac{a^{2}+27 b^{2}}{4}$; en choisissant $b>0, a \equiv 1$ (3) si $m \equiv 1$ (3) , $a=3 a^{\prime}, a^{\prime} \equiv 1$ (3) sinon, le couple $(a, b)$ est associé à un corps $\mathrm{K}$ unique et réciproquement.

Soit E le groupe des unités de norme 1 de $K$; soit $j=\frac{-1+\sqrt{-3}}{2}$; $\mathrm{E}$ est un $\mathbf{Z}[\mathrm{j}]$-module libre de dimension 1 , l'opération étant définie par $\psi^{j}=\psi^{\sigma}, \psi \in E$. Soit $\varepsilon$ un générateur de $E$. Soit $E^{\prime}$ le groupe des unités cyclotomiques de norme 1 de $K$; C'est un sous $-\mathbf{Z}[j]$-module dont on notera $\eta=\epsilon^{\lambda+\mu \sigma} ; \lambda, \mu \in \mathbf{Z}$ un générateur. On sait que $K$ se plonge dans $Q^{(m)}$ et que $\eta$ s'obtient comme norme relative de l'unité cyclotomique de $\Phi^{(m)}$ ([4]). 
Pour toute unité $\psi \in E$, on notera $R(\psi)$ le régulateur de $\psi$. On sait que le nombre de classes $h$ de $K$ est égal à l'indice de $E^{\prime}$ dans $E$, c'est-à-dire que $h=\frac{R(\eta)}{R(\varepsilon)}=\lambda^{2}-\lambda \mu+\mu^{2}$.

II. NOMBRE DE CLASSES ET UNITES.

a) Détermination de $E^{\prime}$.

Soit $\mathrm{m}=\mathrm{p}_{1} \ldots \mathrm{p}_{\mathrm{n}}$; il y a $2^{\mathrm{n}-1}$ corps cubiques $\mathrm{K}$ de conducteur $\mathrm{m}$ et on peut représenter les $2^{\mathrm{n}-1}$ groupes de Galois des extensions $\Phi^{(\mathrm{m})} / \mathrm{K}$ par les $2^{n-1}$ groupes d'entiers modulo $m$ suivants :

$$
\mathrm{H}=\mathrm{g}^{3} \times\left\langle\mathrm{g}_{1} \mathrm{~g}_{2}^{\mathrm{r}_{1}}, \mathrm{~g}_{2} \mathrm{~g}_{3}^{\mathrm{r}_{2}}, \ldots, \mathrm{g}_{\mathrm{n}-1} \mathrm{~g}_{\mathrm{n}}^{\mathrm{r}}{ }^{\mathrm{n}-1}\right\rangle ; \text { où }
$$

$g_{i}$ est d'ordre $\varphi\left(p_{i}\right)$ modulo $m$ ( $\varphi$ : fonction d'Euler $)$,

$g_{i} \equiv 1$ modulo $\prod_{j \neq i} p_{j}$,

$r_{i}=1$ ou 2 ,

et où $q=\left\{\prod_{i=1}^{n} g_{i}^{x_{i}}, 1 \leq x_{i} \leq \varphi\left(p_{i}\right)\right\}$ représente $\operatorname{Gal}\left(\Phi^{(m)} / \Phi\right)$.

On en déduit numériquement :

(i) les valeurs de a et b associées à chaque corps $\mathrm{K}$;

(ii) un générateur $\eta$ du groupe des unités cyclotomiques de norme 1 de chaque corps K (d'après H. HASSE ([4]): $\eta=\prod_{x \in H^{\prime}} \frac{\sin \pi \frac{g_{1} x}{m}}{\sin \pi \frac{x}{m}}$, où $H^{\prime} \subset H$ correspond à $\left.\operatorname{Gal}\left(Q_{O}^{(\mathrm{m})} / \mathrm{K}\right)\right)$.

b) Majoration du nombre de classes $h$ de $K$.

Théorème 1. Soit $\psi \in E$ et soit $r$ l'indice dans $E$ du sous $\mathbb{Z}[j]$-module engendré par $\psi$; alors

$$
r \leq \frac{16}{3} \frac{R(\psi)}{\log ^{2} \frac{m-3}{3}} .
$$

Corollaire. Le nombre de classes $h$ de $K$ vérifie l'inégalité :

$$
h \leq \frac{16}{3} \frac{R(\eta)}{\log ^{2} \frac{m-3}{3}} \text {. }
$$


c) "Dévissage" de $\eta$.

Soit $p$ un nombre premier, soit $f$ son degré résiduel dans $Q(j) / Q$ et soit $q=p^{f}$; nous rappelons que $p \mid h$ si et seulement si $q \mid h$. Si $q>\frac{16}{3} R(\eta) / \log ^{2} \frac{m-3}{3}$, alors $p$ ne divise pas $h ;$ sinon, soient $\omega$ et $\omega^{\prime}$ deux entiers de $\Phi(j)$, complexes conjugués, de norme q (si $\left.f=2, \omega=\omega^{\prime}=p\right)$; une méthode nous permet de tester numériquement s'il existe une unité $\varphi \in E$ telle que $n=\varphi^{\prime \prime}\left(\omega^{\prime \prime}=\omega\right.$ ou $\left.\omega^{\prime}\right)$ : dans ce cas $q$ divise $h ;$ sinon $q$ ne divise pas $h$. Lorsque $q$ divise $h$, la recherche des diviseurs de $h / q$ se fait au moyen du même critère, à partir de l'unité $\varphi$ et du majorant $\frac{16}{3} R(\varphi) / \log ^{2} \frac{m-3}{3}$. Cette méthode permet de déterminer tous les diviseurs premiers de $h$ avec leur ordre de multiplicité. Un générateur $\epsilon$ de $E$ est la dernière unité $\varphi$ obtenue.

d) Relation entre les "classes ambiges" de $K$.

La connaissance d'un générateur $\epsilon$ de $E$ permet de déterminer la relation entre les "classes ambiges" de $K$ (cf. [1]). En effet, d'après le théorème 90 de Hilbert, $\epsilon=\rho^{1-\sigma}, \rho=1+\epsilon+\epsilon \epsilon^{\sigma} ;(\rho)$ est un idéal ambige qui peut donc s'écrire $(p)=q \prod p_{i}, q \in Q, p_{i}$ idéal 1 er au-dessus de $p_{i}$; les $x_{i}$ ne sont pas tous congrus à zéro modulo 3 , sinon $\epsilon$ ne serait pas un générateur de $\mathrm{E}$; ceci donne la relation cherchée.

\section{e) Résultats numériques.}

Nous avons établi des tables donnant le nombre de classes de tous les corps cubiques cycliques de conducteur $\mathrm{m}<4000$, ainsi que des tables partielles, pour $4000<\mathrm{m}<20000$. Donnons un aperçu des résultats obtenus :

(i) Il y a 630 corps cubiques cycliques de conducteur $\mathrm{m}<4000$; parmi eux, il y a :

272 corps de conducteur $m=p_{1}$ (alors $h \equiv 1(3)$ ) et $h=1$ pour 230 d'entre eux,

310 corps de conducteur $\mathrm{m}=\mathrm{p}_{1} \mathrm{p}_{2}$ (alors $\mathrm{h} \equiv 0(3)$ ) et $\mathrm{h}=3$ pour 249 d'entre eux,

48 corps de conducteur $m=p_{1} p_{2} p_{3}$ (alors $h \equiv 0(9)$ ) et $h=9$ pour 43 d'entre eux. 
(ii) Les corps dont un générateur $\epsilon$ de $E$ est totalement positif sont assez rares. Il $y$ en a cinq pour des conducteurs $m<4000$ : ce sont les corps définis par: $m=19.37(a=25, b=9), m=9.79 \quad(a=12, b=10)$, $\mathrm{m}=1009, \mathrm{~m}=1699$ et $\mathrm{m}=31.103(\mathrm{a}=55, \mathrm{~b}=19)$.

(iii) Exemples :

a) $\mathrm{m}=4867=31.157, \mathrm{a}=136, \mathrm{~b}=6 ; \frac{16}{3} \mathrm{R}(\eta) / \log ^{2} \frac{\mathrm{m}-3}{3}$ a pour partie entière $942 ; \mathrm{h}=228=3.4 \cdot 19$ et $\eta=\epsilon^{-14+2 \sigma}$.
b) $m=7351$
$\mathrm{h}=49$
$\eta=\epsilon^{(3+2 \sigma)^{2}}$
$\mathrm{m}=8563$
$h=49$
$\eta=\epsilon^{(3+2 \sigma)^{2}}$
$\mathrm{m}=10267$
$h=49$
$\eta=\epsilon^{7}$
$m=18367$
$h=49$
$\eta=\epsilon^{(3+2 \sigma)^{2}}$.

II. EXISTENCE DES Z-BASES D'ENTIERS DE LA FORME $\left\{1, \vartheta, \vartheta^{2}\right\}$.

L'anneau des entiers A d'un corps cubique sera dit monogène s'il existe une $\mathbf{Z}$-base d'entiers de la forme $\left\{1, \vartheta, \vartheta^{2}\right\}([5])$.

a) Principaux résultats. (Pour les démonstrations se reporter à [3]).

Théorème 2. Soit $K$ un corps cubique cyclique de conducteur $m=\frac{a^{2}+27 b^{2}}{4}$. L'anneau des entiers de $K$ est monogène si et seulement si l'équation diophantienne en $u, v \in \mathbb{Z}$,

$$
b u\left(u^{2}-9 v^{2}\right)+a v\left(u^{2}-v^{2}\right)=1
$$

admet une solution.

Théorème 3 . Soit $K$ un corps cubique cyclique de conducteur $\mathrm{m}$. L'anneau des entiers de $K$ est monogène si et seulement si $K$ possède une unité $w$ de norme 1 vérifiant les conditions :

(i) $\operatorname{Tr}_{K / Q}\left(\mathrm{w}+\mathrm{w}^{-1}\right)=3$,

(ii) $\operatorname{Tr}_{K / Q}\left(\frac{w^{2}-w^{-1}}{m}\right)$ est le cube d'un entier rationnel $Y$. 
Théorème 4. Soit $\mathrm{m}$ un conducteur ; il existe un corps $\mathrm{K}$ cubique cyclique de conducteur $\mathrm{m}$ dont l'anneau des entiers est monogène, si et seulement si $\mathrm{m}$ est de la forme $m=\frac{\alpha^{2}+27}{4 \gamma^{3}}, \alpha, \gamma \in \mathbb{Z}, \alpha \neq \equiv \pm 9$ (27). Lorsque cette condition est réalisée, $K$ est le corps de décomposition du polynome $X^{3}-\frac{\alpha-3}{2} X^{2}-\frac{\alpha+3}{2} X-1$.

Conditions nécessaires pour que A soit monogène. On définit le corps $\mathrm{K}$ par $a$ et $b \quad\left(m=\frac{a^{2}+27 b^{2}}{4}\right)$ et, si $\epsilon$ est un 'générateur de $E$, on pose $\left.\mathrm{t}=\operatorname{Tr}_{\mathrm{K} / \Phi^{(\epsilon)}}, \mathrm{s}=\operatorname{Tr}_{\mathrm{K} / \Phi^{(\epsilon}}{ }^{-1}\right)$. Chacune des conditions suivantes est une condition nécessaire de monogénéité (cf. [5] pour $1,3,4$ ) :

1) $\mathrm{a}$ et $\mathrm{b}$ sont impairs ;

2) si $a \equiv 0(3)$, alors $b \equiv \pm 1 \quad(9) \quad(b>0)$, si $b \equiv 0$ (3), alors $a \equiv 1$ (9) $(a \equiv 1(3))$;

3) la relation entre les idéaux ambiges de $\mathrm{K}$ est $p_{1} \ldots \mathrm{p}_{\mathrm{n}} \sim(1)$;

4) $\epsilon$ non totalement positif ;

5) $\mathrm{s}+\mathrm{t}$ est impair ;

6) $\mathrm{s}+\mathrm{t}+3 \equiv 0(\mathrm{~m})$.

b) Résultats numériques.

Les résultats numériques proviennent essentiellement de l'étude de l'équation diophantienne

$$
b u\left(u^{2}-9 v^{2}\right)+a v\left(u^{2}-v^{2}\right)=1
$$

En recherchant des formes de $a$ et $b$ pour lesquels cette équation admet nécessairement une solution en $(u, v)$, on démontre le résultat suivant :

Proposition. Les corps cubiques cycliques dont le conducteur $m$ est de la forme $\frac{\left(b^{2}-8\right)^{2}+27 b^{2}}{4}$ ou $m=\frac{\left(1-8 b^{2}\right)^{2}+27 b^{2}}{4}, b$ impair admettent une $z$-base d'entiers $\left\{1, \vartheta, \vartheta^{2}\right\}$.

En se donnant $\mathrm{u}$ et $\mathrm{v}$ et en cherchant si l'équation (1) admet des solutions en $a$ et $b$ telles que $m=\frac{a^{2}+27 b^{2}}{4}$ soit un conducteur, on obtient un procédé qui nous a permis de trouver 24 corps de conducteur $\mathrm{m}<10000$, admettant une base d'entiers de la forme $\left\{1, \vartheta, \vartheta^{2} ;\right.$ et autres que les 101 corps dont le conducteur est de la forme $\frac{a^{2}+27}{4}$ ou $\frac{1+27 b^{2}}{4}$, lesquels admettent trivialement une telle base. Donnons la liste de ces 24 corps qui ont été obtenus 
en faisant parcourir à $u$ et $v$ les valeurs entières telles que $u^{2}+3 v^{2}$ soit inférieur à 100000 .

\begin{tabular}{|c|c|c|c|r|r|r|r|r|}
\hline $\mathrm{m}$ & $\mathrm{a}$ & $\mathrm{b}$ & $\mathrm{m}$ & $\mathrm{a}$ & $\mathrm{b}$ & \multicolumn{1}{c|}{$\mathrm{m}$} & $\mathrm{a}$ & $\mathrm{b}$ \\
\hline 241 & -17 & 5 & 1381 & 31 & 13 & 31.163 & -77 & 23 \\
373 & 13 & 7 & 7.229 & 43 & 13 & 9.613 & 111 & 9 \\
379 & -29 & 5 & 1879 & 73 & 9 & 13.457 & -83 & 25 \\
463 & -23 & 7 & 2539 & -83 & 11 & 7.883 & -71 & 27 \\
751 & -41 & 7 & 9.307 & 57 & 17 & 7621 & -161 & 13 \\
19.61 & 37 & 11 & 2797 & -89 & 11 & 7.31 .37 & 97 & 29 \\
1213 & -17 & 13 & 19.211 & -113 & 11 & 7.13 .97 & -125 & 27 \\
1321 & -71 & 3 & 37.109 & 43 & 23 & 13.19 .37 & 103 & 31 \\
\hline
\end{tabular}

Nous ne savons pas s'il existe d'autres corps de conducteur $\mathrm{m}<10000$ et admettant une $\mathbb{Z}$-base d'entiers de la forme $\left\{1, \vartheta, \vartheta^{2}\right\}$. Une telle étude ne peut se faire qu'en étudiant l'équation (1) au moyen des méthodes de Baker.

-:-:-:-

BIBLIOGRAPHIE

[1] - C. CheValley - La théorie du corps de classes dans les corps finis et les corps locaux (Thèse), Journal of the Faculty of Science, Tokyo (1933).

[2] - M.N. GRAS - Méthodes et algorithmes pour le calcul numérique du nombre de classes et des unités des extensions cubiques cycliques de $Q$ (à paraftre au Journal de Crelle).

[3] - M.N. GRAS - Extensions cubiques cycliques de $\mathbb{Q}$ dont l'anneau des entiers est monogène. Ann. Fac. Sc., Besançon, 1973.

[4] - H. HASSE - Über die Klassenzahl abelscher Zahlkorpern, Chapitre I et II, Berlin (1952).

[5] - J.J. PAYAN - Sur les classes ambiges et les ordres monogènes d'une extension cyclique de degré premier impair sur $Q$ ou sur un corps quadratique imaginaire (à paraftre à Arkiv för mathematik).

$-:-:-:-$

Marie-Nicole GRAS

Institut de Mathématiques Pures

Laboratoire associé au CNRS n.188

BP 11638402 - ST.MARTIN D'HERES 